\title{
Ações educativas para prevenção de quedas de pacientes hospitalizados: Revisão
}

\section{integrativa}

\author{
Educational actions for the prevention of falls in hospitalized patients: Integrative review \\ Acciones aducativas para la prevención de caídas en pacientes hospitalizados: Revisión integrativa
}

Recebido: 16/03/2021 | Revisado: 22/03/2021 | Aceito: 29/03/2021 | Publicado: 08/04/2021

Vivian Lemes Lobo Bittencourt

ORCID: https://orcid.org/0000-0003-1488-0611

Universidade Regional Integrada do Alto Uruguai e das Missões, Brasil

E-mail: vivillobo@hotmail.com

Sandra da Silva Kinalski

ORCID: https://orcid.org/0000-0002-4841-2288

Universidade Regional do Noroeste do Estado do Rio Grande do Sul, Brasil

E-mail: sandrakinalski@yahoo.com.br

Eniva Miladi Fernandes Stumm

ORCID: https://orcid.org/0000-0001-6169-0453

Universidade Regional do Noroeste do Estado do Rio Grande do Sul, Brasil

E-mail: eniva@unijui.edu.br

Maria Simone Vione Schwengber

ORCID: https://orcid.org/0000-0002-3583-1408

Universidade Regional do Noroeste do Estado do Rio Grande do Sul, Brasil

E-mail: simone@unijui.edu.br

\begin{abstract}
Resumo
Este artigo objetiva analisar evidências sobre ações educativas direcionadas à prevenção de quedas de pacientes no âmbito hospitalar. Trata-se de uma revisão integrativa que incluiu estudos que realizaram ações educativas direcionadas a pacientes, familiares e à equipe assistencial hospitalar com vistas à prevenção de quedas. Busca realizada nas bases de dados: biblioteca virtual em saúde, PubMed e Scopus, publicados entre 2005 e 2020. Compuseram o corpus de análise 19 artigos. Em sua maioria, as evidências dos artigos referem ações educativas direcionadas ao paciente, aos familiares e/ou à equipe assistencial com várias estratégias na perspectiva de prevenir quedas, contudo os estudos que utilizaram abordagens mistas obtiveram resultados mais contundentes. A análise indicou que a inserção do paciente é fundamental quando se pensa em ações educativas com vistas à redução de quedas no âmbito hospitalar. Para que ações educativas sejam exitosas, os pacientes igualmente necessitam de maior aporte de conhecimento, aptos para identificar fatores de risco no referido ambiente e, a partir disso, ter condições de sugerir, juntamente com familiares e equipes, ações prioritárias de redução dos mesmos.
\end{abstract}

Palavras-chave: Educação em saúde; Pacientes; Enfermagem; Acidentes por quedas; Hospitalização.

\begin{abstract}
This article aims to analyze evidence on educational actions aimed at preventing falls from patients in the hospital environment. It is an integrative review that included studies that carried out educational actions directed at patients, family members and the hospital assistance team with a viem to preventing falls. Search performed in the databases: virtual health library, PubMed and Scopus, published between 2005 and 2020. The corpus of analysis comprised 19 articles. Most of the evidence in the articles refers to educational actions directed at the patient, Family members and/or the assistance team with various strategies in order to prevent falls, however studies that used mixed approaches obtained more striking results. The analysis indicated that the insertion of the patient is fundamental when thinking about educational actions with a view to reducing falls in the hospital environment. For educational actions to be successful, patients also need a greater contribution of knowledge, able to identify risk factors in that environment and, from that, be able to suggest, together with Family members and teams, priority actions to reduce them.
\end{abstract}

Keywords: Health education; Patients; Nursing; Accidental falls; Hospitalization.

\section{Resumen}

Este artículo tiene como objetivo analizar la evidencia sobre acciones educativas encaminadas a prevenir caídas de pacientes en el ámbito hospitalario. Se trata de una revisión integradora que incluyó estudios que realizaron acciones educativas dirigidas a pacientes, familiares y equipo de atención hospitalaria con miras a prevenir caídas. Búsqueda realizada em las bases de datos: biblioteca virtual en salud, PubMed y Scopus, publicadas entre 2005 y 2020. El 
corpus de análisis estuvo compuesto por 19 artículos. La mayor parte de la evidencia em los artículos se refiere a acciones educativas dirigidas al paciente, familiares y/o equipo asistencial con diversas estratégias para prevenir caídas, sin embargo los estudios que utilizaron abordajes mixtos obtuvieron resultados más llamativos. El análisis indicó que la inserción del paciente es fundamental a la hora de pensar en acciones educativas con miras a reducir las caídas en el ámbito hospitalario. Para que las acciones educativas tengan éxito, los pacientes también necesitan un mayor aporte de conocimientos, capaces de identificar los factores de riesgo en esse entorno y, a partir de ello, poder sugerirm junto a familiares y equipos, acciones prioritárias para reducirlos.

Palabras clave: Educación en Salud; Pacientes; Enfermería; Accidentes por caídas; Hospitalización.

\section{Introdução}

As quedas são um problema global de saúde pública. Estima-se que 646.000 quedas fatais ocorrem anualmente, o que as tornam a segunda principal causa de morte por lesão não intencional. Embora não sejam fatais, a cada ano cerca de 37,3 milhões de quedas são graves e requerem atenção da equipe de saúde (WHO, 2018). Situações em que os pacientes caem e sofrem lesão são importantes e permanecem entre os eventos hospitalares adversos mais relatáveis em âmbito mundial (Quigley, 2016). Em 2013, o Ministério da Saúde brasileiro publicou a portaria 529, que instituiu o Programa Nacional de Segurança do Paciente e essa determinação reforçou para as instituições de saúde, os profissionais e a comunidade a importância de abordarem e promoverem ações de segurança do paciente, com foco nas seis metas definidas, dentre as quais destaca-se a prevenção de quedas (Brasil, 2013a).

A queda, além de ser considerada um risco com fatores predisponentes amplos, Barbosa et al. (2019) e Luzia et al. (2019) apresentam relatos de complicações relacionadas especificamente à área hospitalar. Luzia et al. (2019) concluíram que pacientes hospitalizados, que apresentaram queda com dano, eram na sua maioria mulheres (55\%), com idade média de $73 \pm 11,6$ anos, internadas para tratamento clínico (68\%). As quedas ocorreram da própria altura $(63,4 \%)$ e no quarto da(o) paciente $(67,3 \%)$, com gravidade dos danos leve (escoriações) em 80,8\% dos casos, grave (fraturas, alterações de estado mental) em 11,9\% e moderado (ferimento, traumatismo cranioencefálico leve) em 7,3\%. A utilização de estratégias para redução de danos decorrentes de quedas pelos profissionais que cuidam é importante, cientes dos fatores intrínsecos, extrínsecos e comportamentais do paciente que contribuem para a ocorrência do evento. Assim, garantir um atendimento seguro e de qualidade para os pacientes hospitalizados é uma das principais metas da assistência hospitalar, para melhorar ações de cuidado, que perpassam pela educação (Ribeiro et al., 2016).

Um estudo mexicano concluiu que a grande maioria $(91,7 \%)$ das atividades de prevenção de quedas para pacientes adultos devem identificar comportamentos e fatores que afetam o risco de quedas; 8,3\% devem identificar as características do ambiente que potencializam a probabilidade de quedas; e a educação de familiares sobre fatores de risco para quedas (Hernández-Herrera et al., 2017). Ressalta-se que o referido estudo não explicita as metodologias utilizadas nas ações educativas.

Ações educativas em saúde compreendem o desenvolvimento de ações direcionadas à educação em saúde que decorre de processo dialético de viver a doença, do indivíduo ao coletivo, pautadas em relações horizontais nas quais pacientes são sujeitos ativos no processo de construção do conhecimento (Vasconcelos, 2001; 2019). Nesse ínterim, ao ampliar conhecimentos sobre prevenção de quedas, emergem vários questionamentos sobre fatores de risco envolvidos na queda e que requerem conscientização do paciente aliados a metodologias nas ações educativas, com vistas a mobilizar pensamentos e atitudes da equipe de enfermagem e dos pacientes sobre esse tema.

Essas inquietações podem ser sanadas de maneira a garantir uma abordagem assertiva, que contribua para o entendimento do paciente sobre o conteúdo abordado e salvaguarde sua segurança enquanto paciente no hospital, extensivo a seu domicílio. Nesse sentido, o Protocolo de Prevenção de Quedas, instituído pelo Ministério da Saúde brasileiro, aponta a necessidade de intervenções multicomponentes e determina ações e intervenções como estratégias de educação de pacientes, 
familiares e estimula ações desde a admissão do paciente até a alta hospitalar, com o uso de materiais educativos (BRASIL, 2013b).

Paiva et al. (2017) analisaram resultados de pesquisas qualitativas sobre percepções de pacientes referentes a medidas que contribuem para sua segurança. Eles concluíram que existem lacunas entre entendimento e envolvimento dos pacientes nesse processo, aliadas à implementação de ações que favoreçam uma comunicação eficaz, a relação profissionais-pacientes, o acesso a informações com interação e que contribuam para uma assistência segura. Vaccari et al. (2016) reportam a atuação da equipe de enfermagem, aliada a estratégias com o uso de abordagens diferenciadas, a fim de conscientizar pacientes quanto ao risco de queda intra-hospitalar e estimular a coparticipação deles e de seus familiares, com vistas a ampliação de atitudes seguras. A partir dessas considerações, o desejo de construir essa revisão integrativa dá-se com o intuito de analisar evidências sobre ações educativas direcionadas à prevenção de quedas de pacientes no âmbito hospitalar.

\section{Metodologia}

Trata-se de uma pesquisa quantitativa, revisão integrativa da literatura conduzida em seis etapas: 1) identificação do tema e seleção da questão de pesquisa; 2) estabelecimento dos critérios de inclusão e exclusão; 3) identificação dos estudos pré-selecionados e selecionados; 4) avaliação dos estudos selecionados; 5) análise e interpretação dos resultados; e 6) apresentação da revisão/síntese do conhecimento (Mendes; Sileira; Galvão, 2008) (Pereira et al., 2018). Inicialmente foi desenvolvido um protocolo de revisão com vistas a reduzir a possibilidade de vieses e garantir a reprodutibilidade (Paula; Padoin; Galvão, 2016).

A escolha pela temática (etapa 1) ocorreu por se tratar de inquietação da autora em reconhecer as ações educativas utilizadas na prevenção de quedas e a elaboração da questão de revisão foi inspirada na estrutura PICo (População, Interesse, Contexto) (Nobre; Bernardo; Jatene, 2004). A questão norteadora dessa revisão foi: quais as evidências científicas nacionais e internacionais relacionadas ao paciente $(\mathrm{P})$ acerca das ações educativas de enfermagem para a prevenção de quedas (I) no hospital (Co)?

Esta revisão integrativa incluiu estudos com pacientes que participaram de ações educativas para prevenção de quedas vinculada à hospitalização, independentemente da metodologia utilizada. Como critérios de inclusão (etapa 2), estabeleceramse: estudos primários; publicados em inglês, português ou espanhol; que envolvessem ações educativas acerca da prevenção de quedas e que respondessem à questão de revisão. Foi determinado recorte temporal de janeiro de 2005 a junho de 2020, visto que a prevenção de quedas foi estabelecida como uma meta prioritária, em 2004, pela Aliança Mundial para a Segurança do Paciente e permanece como uma temática relevante (WHO, 2004). Os critérios de exclusão foram: estudos voltados para outros eixos relacionados à segurança de pacientes; e pesquisas com a população menor de 18 anos.

A busca foi desenvolvida em junho de 2020, de maneira independente, por duas revisoras. As revisoras seguiram os critérios preestabelecidos para inclusão e exclusão das produções, a partir da leitura dos títulos e resumos das produções localizadas em cada base de dados. Posteriormente, foi realizada reunião de consenso e, desse modo, selecionados os artigos para leitura na íntegra. Não ocorreram divergências entre as revisoras quanto à elegibilidade dos mesmos.

A partir da pergunta de pesquisa e do objetivo, foram definidos os Descritores em Ciências da Saúde, MeSH Database e palavras-chave: pacientes, cliente, doente, educação em saúde, educação de pacientes como assunto, enfermagem, prospecto para educação de pacientes, assistência centrada no paciente, prevenção de acidentes, acidentes por quedas, ações educativas, educação para prevenção de quedas, risco de quedas, prevenção de quedas, redução de quedas, programa de educação do paciente, hospitalização, hospital, internação hospitalar. Foi utilizado o operador booleano OR entre eles e entre os acrônimos utilizou-se o operador booleano AND. Para localização dos estudos primários, as buscas foram realizadas nas bases de dados 
eletrônicas Biblioteca Virtual em Saúde (BVS), portal PubMed e Scopus. A figura 1 descreve o fluxograma da seleção dos artigos para a revisão integrativa, baseado no modelo PRISMA.

Figura 1 - Fluxograma do processo de seleção dos estudos segundo o PRISMA. Santo Ângelo, RS, Brasil, 2021. (n=19).
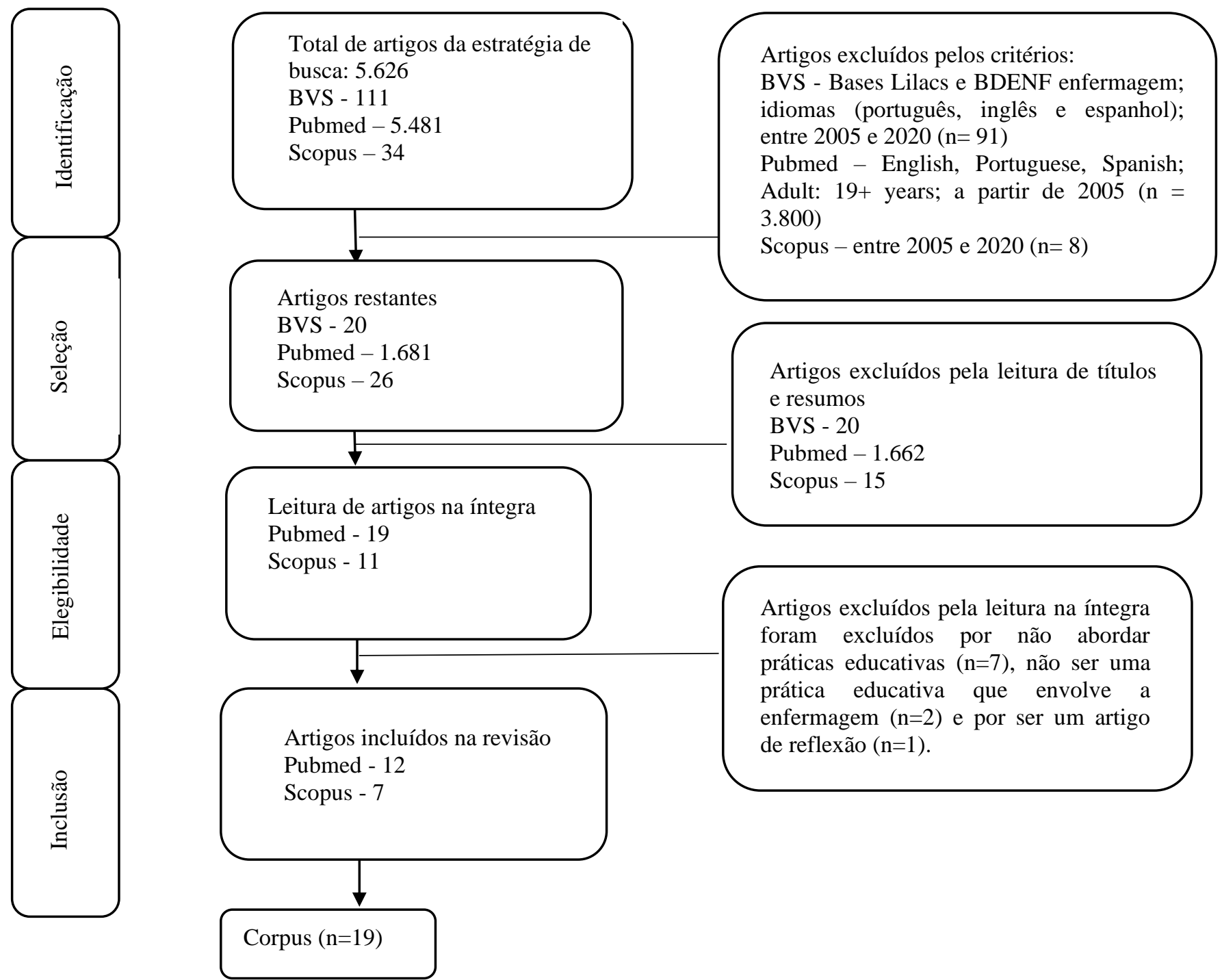

Fonte: Autores.

Para a identificação dos estudos pré-selecionados e selecionados (etapa 3) foi realizada a leitura dos títulos e resumos. A extração dos dados foi desenvolvida em uma planilha no Microsoft Excel com as informações: título, periódico, autor principal, ano de publicação, objetivo, delineamento (abordagem, participantes), nível de evidência e ação educativa implementada para a prevenção de quedas.

Após, realizou-se a análise crítica dos estudos (etapa 4), com agrupamento das informações para a discussão dos resultados. Para avaliação dos estudos foi utilizado um sistema de classificação de força de evidências (Fineout-Overholt; Stillwell, 2011). A interpretação dos resultados (etapa 5) foi pautada na resposta da pergunta de revisão e os resultados dos estudos selecionados foram analisados e discutidos, possibilitando a apresentação (etapa 6) pela similaridade das características. Para identificar os artigos, utilizou-se a nomenclatura "E" referente ao "estudo", seguido do número arábico estabelecido de acordo com o ano de publicação, do mais recente ao mais antigo. Do ponto de vista ético, respeitou-se o direito autoral dos estudos analisados. 


\section{Resultados e Discussão}

Foram selecionados dezenove artigos para análise dos dados e sintetização do conhecimento. A caracterização dos estudos que compuseram o corpus dessa revisão apontou que foram desenvolvidos nos Estados Unidos da América (n=9), na Austrália $(n=2)$ e, com a produção de um estudo por país, no Reino Unido, Espanha, Canadá, Brasil, República da Coreia, Eslovênia, Suíça e Alemanha.

Quanto aos participantes dos estudos identificou-se que eram enfermeiros, médicos, bioestatísticos e fisioterapeutas. O estudo brasileiro foi publicado em português e inglês e o estudo espanhol foi publicado em espanhol e inglês. Os demais estudos $(n=17)$ foram publicados em inglês. O ano de publicação variou de 2006 a 2020, com predomínio de estudos publicados nos anos de 2015 ( $n=4)$ e 2016 ( $n=4)$.

Em relação aos periódicos onde os artigos foram publicados, ocorreu o predomínio de indexações no Journal of Nursing Care Quality ( $\mathrm{n}=5$ ). Com relação a classificação na plataforma Sucupira, de acordo com o quadriênio 2013-2016, na área de avaliação da enfermagem a classificação A1 $(n=10)$ e A2 $(n=5)$, na área de avaliação saúde coletiva a classificação A2 $(\mathrm{n}=1)$ e três periódicos não possuem cadastro na plataforma.

A análise dos artigos indicou que as evidências disponíveis na literatura reportam a ações educativas direcionadas a pacientes, familiares e equipe de saúde para a prevenção de quedas; utilização da avaliação de risco de quedas; atenção com fatores intrínsecos e extrínsecos para promover a prevenção da ocorrência de quedas; e, utilização de diversas abordagens educativas. No Quadro 1, são apresentadas as características dos artigos selecionados com o código correspondente, título, autoria, nível de evidência de acordo com a questão clínica e principais evidências.

Quadro 1 - Caracterização do perfil dos artigos sobre ações educativas de prevenção de quedas na hospitalização, publicados em periódicos no período de janeiro de 2005 a junho de 2020. Santo Ângelo, RS, Brasil, 2021.

\begin{tabular}{llll}
\hline $\begin{array}{c}\text { Código e referência do } \\
\text { artigo }\end{array}$ & Título do artigo e ano & NE/QC & Principais evidências \\
\hline $\begin{array}{l}\text { E1 } \\
\text { (Yasan; Burton; Tracey, }\end{array}$ & $\begin{array}{l}\text { Nurses' documentation of falls } \\
\text { prevention in a patient centred } \\
\text { care plan in a medical ward }\end{array}$ & $\begin{array}{l}\text { N4/T } \\
\text { 2020) }\end{array}$ & $\begin{array}{l}\text { Pecientes com alto risco de queda participavam } \\
\text { prevenção de quedas pela equipe de diário de genciamento de } \\
\text { enfermagem. }\end{array}$ \\
& & $\begin{array}{l}\text { - Estratégias de prevenção de quedas foram: } \\
\text { sinais de alerta de queda acima da cama dos } \\
\text { pacientes, uso de camas baixas, supervisão dos } \\
\text { pacientes no banheiro e uso de campainha nas } \\
\text { camas. }\end{array}$ \\
\end{tabular}

E2

(Radecki;

Kara, 2018)

Reynolds;

Inpatient fall prevention from the patient's perspective: A qualitative study

E3

(Luzia et al., 2018)

E4

(Saiz-Vinuessa

2016)
Incidência de quedas e ações preventivas em um Hospital Universitário

Implantación de una guía de buenas prácticas para la prevención de caídas: percepción de los pacientes hospitalizados y sus cuidadores
$\mathrm{N} 2 / \mathrm{T}$

- A instituição examinou todos os pacientes internados quanto ao risco de queda na admissão e em cada turno.

- Além das medidas universais de prevenção de riscos de queda foram realizadas entrevistas para conscientização

$\mathrm{N} 4 / \mathrm{P}$

- Ações desenvolvidas entre 2011 e 2015 para prevenção de quedas: treinamentos, fôlderes, implantação do uso de pulseiras e cartazes para a sensibilização da equipe, pacientes e familiares. para a prevenção de quedas com avaliação do risco de quedas do paciente nas primeiras 24 horas, aplicação de um plano de cuidados com base em fatores extrínsecos e intrínsecos.

- Educação em saúde com abordagem de dieta adequada, estilo de vida, exercícios, equilíbrio e 
E5

(Opsahl et al., 2017)

E6 (Kuhlenschmidt et al.,
2016)

E7

(Goljar; Globokar; Puzic; Kopitar, 2016)

E8

(Hill et al, 2015)

E9

(Cangany et al, 2015)

E10 (Heck; Gebhart; Gaehle, Tools for a fall-free zone

2015)
Outcomes of Adding Patient and Family Engagement Education

to Fall Prevention Bundled

Interventions

Tailoring Education to

Perceived Fall Risk in

Hospitalized Patients With

Cancer

Effectiveness of a fall-risk reduction programme for inpatient rehabilitation after stroke

Educators' perspectives about how older hospital patients can engage in a falls prevention education programme: a qualitative process evaluation

Bedside Nurses Leading the Way for Falls prevention: an evidence-based approach

Accountability and teamwork: N4/S

\author{
$\mathrm{N} 2 / \mathrm{T}$
}

$\mathrm{N} 4 / \mathrm{S}$

$\mathrm{N} 3 / \mathrm{T}$

$\mathrm{N} 4 / \mathrm{S}$ 
E13

(Dean, 2012)

\section{E14}

(Zuyev; Benoit; Chang;

Dykes, 2011)

E15

(Ireland et al., 2011)

E16

(Ryu; Roche; Brunton, 2009)

E17

(Renteln-Kruse; Krause, 2007)

E18

(Schwendimann; Bühler; Geest; Milisen, 2006)

E19

(Jeske et al., 2006)
Tailored Prevention of Inpatient N6/T

Reducing falls among older N4/T people in hospital

Designing a Falls Prevention N6/T Strategy That Works

Patient and Family Education N6/T for Fall Prevention

Incidence of In-Hospital Falls in Geriatric Patients Before and After the Introduction of an Interdisciplinary Team-Based Fall-Prevention Intervention

Falls and Consequent Injuries in Hospitalized Patients: Effects of an Interdisciplinary Falls Prevention Program

Partnering With Patients and Families in Designing Visual Cues to Prevent Falls in Hospitalized Elders

$\mathrm{N} 6 / \mathrm{T}$

$\mathrm{N} 4 / \mathrm{S}$ mãos.

- Ficha fornecida com lembretes e um teste administrado no final das sessões.

- Projeto FallSafe com o desenvolvimento de educação em saúde para o paciente em que abordava: verificação dos calçados do paciente; orientações sobre campainha e pertences para deixá-los ao alcance; revisão de medicações; e rotina de uso do banheiro.

- Aplicativo Fall TIPS utilizado com o objetivo de evitar quedas de pacientes; avaliação de rotina dos riscos de queda; e proposição de um plano personalizado, acessível à equipe, pacientes e familiares. - Desenvolvido a partir do aplicativo um cartaz colocado próximo à cama do paciente, plano de assistência e orientação do paciente.

- Precauções universais contra quedas que incluía rastrear pacientes em risco de queda e garantir a avaliação.

- Apresentações online de perguntas e respostas, fichas técnicas, pôsteres, um teste de autoaprendizado e um vídeo para o paciente.

- Equipe desenvolveu a marca estratégica "Não caia nessa".

- Programa de prevenção de quedas foi implementado para educação de pacientes/familiares através de: panfletos; avaliação de risco na admissão e a cada 12 horas ou quando condições físicas/mentais do paciente mudam; adesivo aplicado no paciente com alto risco de quedas e nos registros médicos como lembrete para a equipe; alarmes na cama do paciente; horários de higiene; colocar campainha e itens pessoais ao alcance dos pacientes.

- Folheto para pacientes e cuidadores e verificação do progresso das intervenções.

- Para a equipe foi realizada educação em saúde.

- Utilizadas evidências de um protocolo de prevenção de quedas e achados da literatura.

- Examinado o risco de queda pela enfermagem na admissão, os pacientes considerados em risco foram examinados por um médico e foram implementadas medidas gerais para evitar quedas.

- Equipe de enfermagem, pacientes e familiares elaboraram um cartaz sobre prevenção de quedas. O cartaz "Fique seguro, fique parado, está doente, peça ajuda" era apresentado na admissão do paciente com a discussão sobre pedir ajuda ao levantar-se.

Legenda: (NE) Nível de Evidência; (QC) Questão Clínica; (S) classificação a partir das questões clínicas relacionadas ao significado; (P) classificação a partir das questões clínicas relacionadas a prognóstico ou etiologia; (T) classificação a partir das questões clínicas relacionadas a tratamento ou intervenção. Fonte: Autores. 
Com relação aos níveis de evidência classificados podemos destacar que os artigos se enquadram em três pirâmides de níveis distintos, o que denota que esse tema é explorado com diferentes olhares sobre a verificação de questões clínicas, questões de significado ou compreensão dos sentimentos do paciente e questões relacionadas a prognóstico e etiologia. Predominaram os estudos (E1, E2, E6, E11, E12, E14, E16, E18) nos quais o paciente foi alvo das ações educativas desenvolvidas, já outros mantiveram como público-alvo o paciente e familiares (E4, E5, E7, E13, E15, E19). Ainda, pode-se evidenciar que, além da centralidade no paciente, estudos (E3, E9, E10, E17) abordaram o paciente, seus familiares e equipe multiprofissional, e um deles (E8) foi direcionado para paciente e equipe multiprofissional.

As estratégias utilizadas nas abordagens educativas foram variadas. Ao direcionar-se somente ao paciente, foram empregadas desde estratégias universais, como colocação de sinais de alerta de queda acima dos leitos, uso de camas baixas, supervisão dos pacientes no banheiro, uso de alarme nas camas, plano assistencial e orientação do paciente (E1, E2, E14); entrevista e sessões de educação individual (E2, E12, E16, E18); panfletos e fichas educativas para prevenção de quedas (E6, E12, E16), vídeos (E6) e aplicativos como formas de prevenir quedas e/ou sinalizar o risco (E11, E14).

No entanto, para atingir o paciente e familiares foram desenvolvidas ações para prevenção de quedas, tais como: notificação de familiares e paciente no envolvimento dos cuidados no ambiente hospitalar (E5); instalação de placas em banheiro (E5); demonstração do ajuste da cama e da campainha de emergência perto da cama e no banheiro (E7, E13); vídeo educacional (E5, E15); materiais educacionais, guia de boas práticas, cartaz e folhetos educacionais (E15, E4, E19); alerta para piso escorregadio, importância de calçados não escorregadios e equilíbrio (E7, E13); remoção de objetos próximos à cama (E7); apresentações online, perguntas e respostas (E15); criação de uma marca para a ação educativa com o logo "Não caia nessa" (E15) e "Fique seguro, fique parado, está doente, peça ajuda" (E19).

Foram constatadas ações com público-alvo variado, como pacientes, familiares e equipe, e para cada público participante foram constatadas abordagens diferenciadas nos estudos. Para pacientes e familiares, foram planejados cartazes, folhetos e a implementação do uso de pulseiras (E3, E17); vídeo reencenando uma queda (E9); sinalizações de quedas foram projetadas no teto acima das camas dos pacientes (E9); ações de educação para a segurança com o paciente e familiares durante a visita ao leito (E10); conversas com pacientes e familiares sobre precauções contra quedas (E10). Já para a equipe foram realizadas ações como treinamentos (E3); educação em saúde (E9, E17); para manter o programa de prevenção de quedas foram incluídos dias trimestrais no hospital para lembrar "Sem zona de queda" (E9); e discussão sobre o protocolo de prevenção de quedas existente e as alterações estruturais/processuais (E10).

Para pacientes e equipe, foi identificado um único estudo (E8) que desenvolveu um programa de recuperação segura, com intervenções de educação individualizadas para ambos os públicos. Aos pacientes, ainda, forneceu-se um DVD e uma pasta com materiais para leitura sobre instruções e práticas específicas, como por exemplo, aguardar a equipe antes de ir ao banheiro (E8). A educação individualizada em prevenção de quedas fornece aos pacientes motivação para desenvolver e realizar estratégias comportamentais que reduzam as mesmas, apoiadas pela equipe e ambiente (E8). Os educadores também forneceram subsídios à equipe multidisciplinar para facilitar o apoio ao programa, com feedbacks semanais, sobre as metas estabelecidas pelos pacientes sobre o ambiente da enfermaria em relação à mobilidade segura (E8).

Foi constatado também que os estudos trazem importantes contribuições para o conhecimento científico relacionado ao tema educação para a prevenção de quedas, elencam aspectos gerenciais, cuidados de enfermagem e intervenções diante dos problemas relacionados a quedas. Além disso, apesar da maioria $(89,5 \%)$ dos estudos ter sido conduzida por enfermeiras, percebemos que o conhecimento produzido é de interesse de toda a comunidade acadêmica e da sociedade em geral.

Das pesquisas que compuseram o corpus dos resultados, $10,5 \%(\mathrm{n}=2)$ não identificaram efeito positivo de ações educativas direcionadas ao paciente (E1, E18), e associaram as conclusões não satisfatórias com a não identificação de alto risco de quedas em todos os pacientes que os possuíam (E1), com o não envolvimento de todos os pacientes na aplicação da 
ação educativa (E1), e com a não diminuição substancial das frequências de quedas e as consequentes lesões após a implementação do programa interdisciplinar de prevenção de quedas (E18).

Em seis artigos (E1, E5, E7, E10, E13, E16) analisados, foram encontradas ações direcionadas a fatores extrínsecos, ao uso do banheiro e campainha com vistas a contribuir para o aprendizado em saúde e à exposição do paciente nessas ações diárias de vida com maior segurança. Outras três pesquisas (E5, E11, E14) utilizaram tecnologias.

Os estudos que constataram ações educativas como benéficas ressaltaram o uso de associação de múltiplas estratégias (E3, E9, E13, E15, E17); trabalho com familiares e paciente para assumirem papel conjunto mais ativo na redução de quedas (E4, E5, E16, E19); trabalho colaborativo da equipe com o paciente com foco comum na conscientização da prevenção de quedas (E6, E7, E8, E10, E19); uso do aplicativo i Engaging e suas abordagens para envolver pacientes nos cuidados de prevenção de quedas (E11); programas centrados no paciente podem reduzir a dependência de alarmes no leito e permitir implementação de estratégias para mitigar fatores de risco (E2, E10).

Os estudos analisados trazem importantes contribuições no sentido de conhecer o panorama atual de publicações produzidas relacionadas às ações educativas para prevenção de quedas de pacientes no ambiente hospitalar. Isso demonstra a tendência que os estudos têm seguido, os pontos de maior relevância para a prevenção de quedas e o planejamento de ações educativas que envolvem o paciente, familiares e equipes.

Uma das primeiras etapas a serem implementadas nas instituições hospitalares é a identificação do risco de queda. Entende-se por fatores de risco os hábitos e comportamentos das pessoas ou exposição ambiental, associada ao aumento da probabilidade de ocorrer uma doença ou evento (Bonita; Beaglehole; Kjellstrom, 2006). As ações educativas favoráveis para a diminuição da ocorrência de eventos adversos na saúde dos pacientes hospitalizados são executadas sobre fatores de risco reversíveis ou modificáveis e, no que tange às quedas, pacientes hospitalizados têm alto risco de quedas, relacionados ao espaço físico desconhecido e ao estado clínico em que se encontram (Bonita; Beaglehole; Kjellstrom, 2006) (Deandrea et al., 2012). Nessa perspectiva, é salutar o envolvimento do paciente e familiares na formulação de estratégias de prevenção e orientação, diante dos fatores de risco existentes e a consciência da importância da inclusão de todos nesse processo (BRASIL, 2013b).

A hospitalização requer adaptação do paciente à estrutura física, rotinas, normas e relacionamento interpessoal. Estas mudanças no cotidiano, associadas aos agravos à saúde, podem determinar diminuição abrupta da autonomia e da funcionalidade do paciente. Pesquisas identificaram a presença de fatores de risco intrínsecos e extrínsecos para quedas no ambiente hospitalar e pontuaram a necessidade da apropriação desses para favorecer o desenvolvimento de ações de educação em saúde, de prevenção de quedas e de qualificação do cuidado (Bittencourt et al., 2017) (Aguiar et al., 2019).

No que tange ao uso de tecnologias, Gottlieb e Makower (2012) se reportam a estratégias de prevenção de quedas com o uso de tecnologias por melhorar potencialmente o cuidado em saúde, com segurança. Programas de prevenção de quedas, com a inclusão de um vídeo educativo para pacientes e familiares, podem resultar em tendências positivas para a diminuição na taxa de quedas de pacientes no âmbito hospitalar. Intervenção educativa com o uso de vídeo oportuniza a participação dos familiares na discussão e educação sobre prevenção de quedas e colabora positivamente com os pacientes e seus familiares. Abbasi et al. (2017) afirmam que a disposição de vídeos contribui na promoção de uma diversidade de situações para os pacientes, familiares e para a equipe de saúde, pela oportunidade de recriar e simular quedas. Esse reencenar retoma o lúdico, favorece a memorização visual, possibilita aprendizado e a associação de prevenção específica.

O uso de logotipos para as campanhas de prevenção de quedas e dicas visuais aplicadas nos prontuários dos pacientes são fundamentais para a aproximação do paciente ao conteúdo teórico, com a utilização de linguagem acessível e com material de fácil compreensão. A utilização de ilustrações coloridas e figuras causam impacto importante na comunicação (Abbasi; Eslami; Mohammadi; Khajouei, 2017). Entendemos que o uso de instrumentos educativos como folder, guias de boas práticas, 
folhetos, placas, fichas educativas, vídeos e aplicativos são crescentes enquanto recursos no processo de educação em saúde e criam novas possibilidades de interação entre enfermeiro, paciente e familiares.

Contudo, somente a criação de instrumentos não garante o alcance da meta de redução da ocorrência de quedas. A educação em saúde é primordial na construção de um elo com pacientes, seus familiares ou com a equipe de saúde, aliada ao diálogo e reforço das orientações técnicas. Hill et al. (2015) pontuam que a educação individualizada pode capacitar a equipe para trabalhar com a prevenção de quedas de pacientes no ambiente hospitalar. Programas individualizados de educação do paciente, combinados com treinamento e feedback para a equipe reduzem quedas de pacientes e com resultados melhores do que programas de intervenção destinados apenas à equipe (France et al., 2017) (Melin, 2018). Nesse sentido, destaca-se a necessidade de a equipe pensar mais sobre maneiras inovadoras de diminuir as quedas de pacientes hospitalizados (Melin, 2018).

Ações educativas no ambiente hospitalar sobre prevenção de quedas perpassam por todos os envolvidos no cuidado. Assim, as ações de identificação dos riscos, trabalho em equipe, engajamento para reduzir o risco de quedas, a comunicação do status do risco de quedas para o paciente e familiares são estratégias a serem utilizadas neste contexo (Melin, 2018) (Hurley et al., 2009). Ao envolver pacientes e familiares em ações educativas, os capacitamos a se envolverem em sua segurança e oferecemos uma parceria valiosa para evitar a ocorrência de eventos adversos (Dupree; Fritz-Campiz; Musheno, 2014). Por vezes, pacientes não acreditam que estão em risco de cair enquanto hospitalizados ou que este ambiente possa lhes propiciar a ocorrência deste evento (Carroll; Dykes; Hurley, 2010). Dados dos estudos reforçam a hipótese de que simplesmente orientar pacientes após a conclusão da avaliação e do plano de risco de queda é insuficiente, os pacientes podem ser envolvidos em todo o processo de prevenção de quedas, o que é fundamental para reduzir as taxas de quedas em hospitais (Duckworth et al., 2019) (Dykes et al., 2012).

Hitcho et al. (2004) se reportam à dificuldade de realizar intervenções multifatoriais complexas devido ao curto tempo que os pacientes permanecem internados no hospital, mas afirmam que é possível uma única intervenção efetiva, com foco na circunstância mais comum na qual as quedas ocorrem. Nesse âmbito, avalia-se que estratégias de prevenção de quedas no ambiente hospitalar podem sim ser exitosas, desde que focadas em atividades associadas com a realidade de cada instituição. Ações educativas podem ser usadas para integrar a prevenção de quedas baseadas em evidências. São estratégias eficazes para a disseminação, permitindo que diversas instituições as escolham como uma modalidade que se encaixa na cultura organizacional e na infraestrutura hospitalar (Duckworth et al., 2019).

A construção desta revisão com ênfase na área da enfermagem, e considerando o vasto campo de conhecimento da pesquisa, pode ser considerada tanto uma limitação quanto uma potencialidade, pelo fato de instigar profissionais e pesquisadores das outras áreas e afins para a construção de mais pesquisas que envolvam essa temática e, dessa forma, ampliar evidências, reduzir a incidência de quedas e qualificar a assistência ao paciente hospitalizado com segurança. Esta revisão apresenta como limitação o fato de ter sido construída a partir da busca e seleção em três bases de dados.

No entanto, a decisão das pesquisadoras de optar por incluir um recorte temporal representa uma potencialidade do estudo, na medida em que fornece uma visão abrangente da utilização de ações educativas após a implementação de uma meta mundial para redução de quedas. Além disso, discussões dessa ordem contribuem para a expansão teórica e metodológica na enfermagem, por apresentar possibilidade de intervenções no ambiente hospitalar, que podem auxiliar pesquisadores na escolha e aplicabilidade de ações educativas preventivas no futuro.

\section{Conclusão}

A inserção do paciente é fundamental quando se pensa em ações educativas com vistas à redução de quedas no âmbito hospitalar. Nesse sentido, a ampliação do conhecimento das equipes que cuidam, mais especificamente da enfermagem, não 
são suficientes. Para que ações e intervenções educativas sejam exitosas, os pacientes igualmente necessitam de maior aporte de conhecimento, aptos para identificar os fatores de risco no referido ambiente e, a partir disso, ter condições de sugerir ações prioritárias de redução dos mesmos, juntamente com familiares e equipes.

Dentre as estratégias utilizadas nos artigos analisados, o uso de tecnologias é fundamental e se constitui em diferencial no que tange à qualidade do cuidado e principalmente a continuidade após a alta hospitalar. A construção desta revisão pode contribuir para instigar as equipes que cuidam, no sentido de implementar programas de formação continuada que contemple pacientes, familiares, equipes e gestores institucionais para a prevenção de quedas no ambiente hospitalar, principalmente como subsídios para a criação de uma cultura organizacional e alcance da meta de redução de quedas de pacientes.

Deixa-se como sugestão para estudos futuros a possibilidade de escrita sobre a temática com recorte temporal maior com o intuito de verificar o que era planejado antes das legislações publicadas sobre segurança do paciente. Outras bases de dados podem ser consultadas com vistas a possibilitar a captura de artigos com outras metodologias e ações educativas direcionadas a prevenção de quedas no ambiente hospitalar.

\section{Referências}

Abbasi, M., Eslami, S., Mohammadi, M. \& Khajouei, R. (2017). The pedagogical effect of a health education application for deaf and hard of hearing students in elementary schools. Electron Physician, 9(9), 5199-5205.

Aguiar, J. R., Barbosa, A. O., Galindo Neto, N. M., Ribeiro, M. A., Caetano, J. Á. \& Barros, L. M (2019). Fatores de risco associados à queda em pacientes internados na clínica médica-cirúrgica. Acta Paul. Enferm. (Online), 32(6), 617-623.

Barbosa, A. S., Chaves, E. H. B., Ribeiro, R. G., Quadros, D. V., Suzuki, L. M. \& Magalhães, A. M. M (2019). Characterization of the adult patients' falling incidents in a university hospital. Rev. gaúcha enferm, 40(n.spe), pe20180303.

Bittencourt, V. L. L., Graube, S. L., Stumm, E. M. F., Battisti, I. D. E., Loro, M. M., \& Winkelmann, E. R. (2017). Factors associated with the risk of falls in hospitalized adult patients. Rev. Esc. Enferm. USP, 51(e03237), 1-7.

Bonita, R., Beaglehole, R. \& Kjellstrom, .T. (2006). Basic epidemiology 2a ed. Geneva: WHO Press, Word Health Organization.

Brasil (2013a). Ministério da Saúde. Portaria n n $^{\text {529, de }} 1^{\text {o }}$ de abril de 2013. Institui o Programa Nacional de Segurança do Paciente (PNSP): Diário Oficial da República Federativa do Brasil.

Brasil (2013b). Ministério da Saúde. Anexo I: protocolo prevenção de quedas. Brasília: Ministério da Saúde, Anvisa, Fio Cruz; 2013.

Cangany, M., Back, D., Hamilton-Kelly, T., Altman, M. \& Lacey, S. (2015). Bedside Nurses Leading the Way for Falls Prevention: An Evidence-Based Approach. Crit. care nurse, 35(2): 82-84.

Carroll, D. L., Dykes, P. C. \& Hurley, A. C. (2010). Patients' perspectives of falling while in an acute care hospital and suggestions for prevention. Appl. nurs. res., 23(4), 238-241.

Clarke, H. D., Timm, V. L., Goldberg, B. R. \& Harttrup, S. J. (2012). Preoperative Patient Education Reduces In-hospital Falls After Total Knee Arthroplasty. Clin. Orthop. relat. res., 470(1), 244-249.

Dean, E. (2012). Reducing falls among older people in hospital. Nurs. older people., 24(5), 6-19.

Deandrea, S., Lucenteforte, E., Bravi, F., Foschi, R., Vecchia, C. L. \& Negri, E. (2010). Risk factors for falls in community-dwelling older people: a systematic review and meta-analysis. Epidemiol. (Baltimore), (21)5, 658-668.

Duckworth, M. et al. (2019). Assessing the Effectiveness of Engaging Patients and Their Families in the Three-Step Fall Prevention Process Across Modalities of an Evidence-Based Fall Prevention Toolkit: An Implementation. Science Study. J. med. internet res., $21(1)$, e10008.

Dupree, E., Fritz-Campiz, A. \& Musheno, D. (2014). A new approach to preventing falls with injuries. J. nurs. care qual., 29(2), 99-102.

Dykes, P. C., I-Ching, E. H., Soukup, J. R., Chang, F. \& Lipsitzet, S. (2012). A Case Control Study to Improve Accuracy of na Electronic Fall Prevention Toolkit. Proc. AMIA Annu. Symp., 170-179.

Fineout-Overholt, E. \& Stillwell, S. B. (2011). Asking compelling, clinical questions. In: B. M. Melnyk, E. Fineout-Overholt. Evidence-based practice in nursing \& healthcare: a guide to best practice. Philadelphia: Wolters Kluwer, Lippincott Williams \& Wilkins, 25-39.

France, D., Slayton, J., Moore, S., Domenico, H., Matthews, J. \& Steban, R. L. et al. (2017). A multicomponent fall prevention strategy reduces falls at an academic medical center. The Joint Commission Journal on Quality and Patient Safety, 43(9), 460-470.

Goljar, N., Globokar, D., Puzic, N. \& Kopitar, N. (2016). Effectiveness of a fall-risk reduction programme for inpatient rehabilitation after stroke. Disabil. rehabil., 38(18),1-9. 
Gottlieb, S. \& Makower, J. (2013). A role for entrepreneurs: an observation on lowering healthcare costs via technology innovation. Am. j. prev. med., 44(1).

Heck, J., Gebhart, A. \& Gaehle, K. (2015). Accountability and teamwork: tools for a fall-free zone. Nurs. manage., 46(1), 40-45.

Hernández-Herrera, D., Aguilera-Elizarraraz, N., Veja-Argote, M., Gonzálesz-Quirarte, N., Castañeda-Hidalgo, H. \& Isase-Hernández, L. (2017). Aplicación de las actividades de la intervención de enfermería Prevención de caídas en adultos hospitalizados. Enferm. univ., 14(2), 118-123.

Hill, A., McPhail, A., Francis-Coad, N., Waldron, N., Erherton-Beer, C. \& Flicker, L., et al. (2015). Educators' perspectives about how older hospital patients can engage in a falls prevention education programme: a qualitative process evaluation. BMJ Open, 5(e009780).

Hill, A., McPhail, A., Waldron, N., Erherton-Beer, A., Ingram, K. \& Flicker, L. et al. (2015). Fall rates in hospital rehabilitation units after individualised patient and staff education programmes: A pragmatic, steppedwedge, cluster-randomised controlled trial. Lancet, 385(9987), 2592-2599.

Hitcho, E. B. et al. (2004). Characteristics and circumstances of falls in a hospital setting: A prospective analysis. J. gen. intern. med. Medicine, 19(7), 732739.

Hurley, A. C., Dyckes, P., Carroll, D. L., Dykes, J. \& Middleton, B. (2009). Fall TIP: Validation of Icons to Communicate Fall Risk Status and Tailored Interventions to Prevent Patient Falls. Studies in Health Technology and Informatics, 146, 455-459.

Ireland, S., Lazar, T., Mayrak, C., Morgan, B., Pizzacalla, A. \& Reis, C., et al. (2010). Designing a Falls Prevention Strategy That Works. J. nurs. care qual., 25(3), 198-207.

Jeske, L., Kolmer, V., Muth, M., Cerns, S., Moldenhaur, S. \& Hook, M.L. (2006). Partnering With Patients and Families in Designing Visual Cues to Prevent Falls in Hospitalized Elders. J. nurs. care qual., 21(3), 236-241.

Kuhlenschmidt, M. L., Reeber, C., Wallace, C., Chen, Y., Barnholtz-Sloan, J. \& Mazanec, S. (2016). Tailoring Education to Perceived Fall Risk in Hospitalized Patients With Cancer: A Randomized, Controlled Trial. Clin. j. oncol. Nurs., 20(1), 84-89.

Luzia, M. F., Cassola, T. P., Suzuki, L. M., Dias, V. L. M., Pinho, L. B. \& Lucena, A. F. (2018). Incidência de caídas y acciones preventivas en un Hospital Universitario. Rev. Esc. Enferm. USP., 52(e03308).

Luzia, M. F., Prates, C. G., Bombardelli, C. F., Adorna, J. B. \& Moura, G. M. S. S. (2019). Characteristics of falls with damage to hospitalized patients. Rev. gaúcha enferm., 40(n.spe), e20180307.

Melin C. (2018). Reducing falls in the inpatient hospital setting. Int. j. evid.-based healthc., 16(1), $25-31$.

Mendes, K. D. S., Silveira, R. C. C. P. \& Galvão, C. M. (2008). Integrative literature review: a research method to incorporate evidence in health care and nursing. Texto Contexto Enferm., 17(4), 758-764.

Nobre, M. R., Bernardo, W. M. \& Jatene, F. B. (2004). Evidence based clinical practice: part I - well structured clinical questions. Rev. bras. reumatol., 44(6), $397-402$.

Opsahl, A. G., Ebright, P., Cangany, M., Lowdwe, M., Scott, D. \& Shaner, T. (2017). Outcomes of Adding Patient and Family Engagement Education to Fall Prevention Bundled Interventions. J. nurs. care qual., 32(3), 252-258.

Paiva, M. C. M. S., Gallasch, C. H., Lima, S. A. M., Sitton-Kent, L., Devo, R. \& Xyrichis, A. (2017). Patients' perceptions of health care-related infections and safety measures. Rev. enferm UERJ, 25(e27468).

Paula, C. C., Padoin, S. M. M. \& Galvão, C. M. (2016). Metodologias da pesquisa para a enfermagem e saúde: da teoria à prática. In: Lacerda LMR, Costenaro RGS, editores. Revisão integrativa como ferramenta para tomada de decisão na prática em saúde. 226 - 230.

Pereira, A. S et al. (2018). Metodologia da pesquisa científica. UFSM.

Quigley, P. A. (2016). Evidence levels: applied to select fall and fall injury prevention practices. Rehabil. nurs., 41(1), 5-15.

Radecki, B., Reynolds, S. \& Kara, A. (2018). Inpatient fall prevention from the patient's perspective: A qualitative study. Appl. nurs. res., 43:114-119.

Renteln-Kruse, W. \& Krause, T. (2007). Incidence of In-Hospital Falls in Geriatric Patients Before and After the Introduction of an Interdisciplinary TeamBased Fall-Prevention Intervention. J. am. geriatr. soc., 55(12), 2068-2074.

Ribeiro, H. C. T. C, Santos, D. S., Paula, A. O., Freire, E. M. R. \& Alves, M. (2016). Non-conformities in hospitals related to the infection and adverse events prevention and control. Rev. enferm. UFPE on line, 10(9), 3344-51.

Ryu, M., Roche, J. P. \& Brunton, M. (2009). Patient and Family Education for Fall Prevention. J. nurs. care qual., 24(3), $243-249$.

Saiz-Vinuessa, M. D., Muñoz-Mansilha, E., Muñoz-Serrano, T., Córcoles-Jiménez, M. P., Ruiz-García, M. V. \& Fernández-Pallarés, P., et al. (2016). Implantación de una guía de buenas prácticas para la prevención de caídas: percepción de los pacientes hospitalizados y sus cuidadores. Rev. calid. asist., 31(6), 329-337.

Schwendimann, R., Bühler, H., Geest, S. \& Milisen, K. (2006). Falls and Consequent Injuries in Hospitalized Patients: Effects of an Interdisciplinary Falls Prevention Program. BMC health serv. res. (Online)., 6(69), 1-7.

Tzeng, H., Yin, C., Fitzgerald, K. \& Graham, K. (2015). i Engaging User Testing Lessons Learned From Inpatients and Health Care Providers. J. nurs. care qual., 30(3), 275-282.

Vaccari, E., Lenardt, M. H., Wilig, M. H., Betiolli, S. E. \& Andrade, L. A. S. (2016). Patient safety and falls in the hospital environment. Rev. cogitare enferm., 21(n. esp), 01-09. 
Research, Society and Development, v. 10, n. 4, e21110413954, 2021

(CC BY 4.0) | ISSN 2525-3409 | DOI: http://dx.doi.org/10.33448/rsd-v10i4.13954

Vasconcelos, E. M. (2001). A saúde nas palavras e nos gestos: reflexões da rede de educação popular e saúde. $1^{\text {a }}$ ed. São Paulo: Hucitec.

Vasconcelos, E. M. (2019). Educação popular em saúde: constituição e transformação de um campo de estudos e práticas na saúde coletiva. In: Pelicioni MCF, Mialh FL, editores. Educação e promoção da saúde: teoria e prática. Rio de Janeiro: Santos.

WHO, World Health Organization. (2018). Fall. Geneva(CH): WHO.

WHO, World Health Organization. (2004). Patient safety: a global priority. Bull World Health Organ.

Yasan, C., Burton, T. \& Tracey, M. (2020). Nurses' documentation of falls prevention in a patient centred care plan in a medical Ward. Aust. j. adv. nurs., $37(2), 19-24$

Zuyev, L., Benoit, A. N., Chang, F. Y. \& Dykes, P. C. (2011). Tailored Prevention of Inpatient Falls. Comput. inform. nurs., 29(2), 93-100. 